\title{
Schichten mit geschätzten Schichtgrößen II
}

(Eine Weiterentwicklung und eine Korrektur zum Artikel des gleichen Autors im Heft 1/1996 der Österreichischen Zeitschrift für Statistik)

\author{
Andreas Quatember \\ Institut für Angewandte Statistik, Universität Linz
}

Quatember (1996) untersucht die statistischen Eigenschaften der Schätzer $\bar{x}$ in geschichteten Zufallsauswahlen. Die Varianz des Schätzers $\bar{x}$ beträgt bei proportionaler Allokation des Stichprobenumfanges auf die Schichten und Ziehen mit Zurücklegen (für die Notationen siehe: Quatember, 1996)

a) bei Verwendung der wahren Schichtanteile $\pi_{\mathrm{i}}(\mathrm{i}=1,2, \ldots, \mathrm{r})$ des Schichtmerkmals für eine geschichtete Zufallsauswahl vom Umfang $n_{S}$ :

$$
\operatorname{Var} \overline{\mathrm{x}}_{\mathrm{S}}=\frac{1}{\mathrm{n}_{\mathrm{S}}} \sum_{\mathrm{i}=1}^{\mathrm{r}} \pi_{\mathrm{i}} \sigma_{\mathrm{i}}^{2}
$$

b) bei Verwendung geschätzter Schichtanteile für eine geschichtete Zufallsauswahl vom Umfang $\mathrm{n}_{\mathrm{gS}}$, wenn die Schätzung der $\mathrm{r}$ Schichtgrößen auf Basis einer uneingeschränkten Zufallsauswahl vom Umfang $\mathrm{n}_{\mathrm{Z}}$ erfolgt ist:

$$
\operatorname{Var} \bar{x}_{g S, Z}=\frac{1}{n_{g S}} \sum_{i=1}^{r} \pi_{i} \sigma_{i}^{2}+\frac{1}{n_{z}} \sum_{i=1}^{r} \pi_{i}\left(\mu_{i}-\mu\right)^{2},
$$

c) bei Verwendung geschätzter Schichtanteile für eine geschichtete Zufallsauswahl vom Umfang $n_{\mathrm{gS}}$, wenn die Schätzung der $\mathrm{r}$ Schichtgrößen auf Basis einer geschichteten Zufallsauswahl vom Umfang $\mathrm{n}_{\mathrm{S}}$ erfolgt ist:

$$
\operatorname{Var} \bar{x}_{\mathrm{gS}, \mathrm{S}}=\frac{1}{\mathrm{n}_{\mathrm{gS}}} \sum_{\mathrm{i}=1}^{\mathrm{r}} \pi_{\mathrm{i}} \sigma_{\mathrm{i}}^{2}+\frac{1}{\mathrm{n}_{\mathrm{S}}}\left(\sum_{\mathrm{i}=1}^{\mathrm{r}} \pi_{\mathrm{i}} \mu_{\mathrm{i}}^{2}-\sum_{\mathrm{k}=1}^{\mathrm{s}}\left(\sum_{\mathrm{i}=1}^{\mathrm{r}} \mu_{\mathrm{i}} \pi_{\mathrm{i} \mid \mathrm{k}}\right)^{2} \frac{\mathrm{N}_{\mathrm{k}}}{\mathrm{N}}\right) .
$$

(3) ist dabei eine Weiterentwicklung der Varianzformel aus Quatember (1996), denn der dort in (9) auf S.51 angegebene Subtrahend

$$
\sum_{\mathrm{i}} \sum_{\mathrm{j}} \mu_{\mathrm{i}} \mu_{\mathrm{j}}\left(\sum_{\mathrm{k}} \frac{\mathrm{N}_{\mathrm{k}}}{\mathrm{N}} \pi_{\mathrm{i} \mid \mathrm{k}} \pi_{\mathrm{j} \mid \mathrm{k}}\right)
$$

läßt sich darstellen als

$$
\begin{aligned}
\sum_{\mathrm{i}} \sum_{\mathrm{j}} \mu_{\mathrm{i}} \mu_{\mathrm{j}}\left(\sum_{\mathrm{k}} \frac{\mathrm{N}_{\mathrm{k}}}{\mathrm{N}} \pi_{\mathrm{i} \mid \mathrm{k}} \pi_{\mathrm{j} \mid \mathrm{k}}\right) & =\sum_{\mathrm{k}} \frac{\mathrm{N}_{\mathrm{k}}}{\mathrm{N}} \sum_{\mathrm{i}} \mu_{\mathrm{i}} \pi_{\mathrm{i} \mid \mathrm{k}} \sum_{\mathrm{j}} \mu_{\mathrm{j}} \pi_{\mathrm{j} \mid \mathrm{k}} \\
& =\sum_{\mathrm{k}}\left(\sum_{\mathrm{i}} \mu_{\mathrm{i}} \pi_{\mathrm{i} \mid \mathrm{k}}\right)^{2} \frac{\mathrm{N}_{\mathrm{k}}}{\mathrm{N}},
\end{aligned}
$$

woraus sich (3) ergibt. 
Der durch diese Vorgangsweise entstehende Schichtungseffekt $\Delta$ (Var) läßt sich mit (3) nun folgendermaßen darstellen:

$$
\begin{aligned}
\Delta(\operatorname{Var}) & =\operatorname{Var} \overline{\mathrm{x}}_{\mathrm{Z}}-\operatorname{Var} \overline{\mathrm{x}}_{\mathrm{gS}, \mathrm{S}} \\
& =\frac{1}{\mathrm{n}_{\mathrm{gS}}} \sum_{\mathrm{i}} \pi_{\mathrm{i}}\left(\mu_{\mathrm{i}}-\mu\right)^{2}-\frac{1}{\mathrm{n}_{\mathrm{S}}}\left(\sum_{\mathrm{i}} \pi_{\mathrm{i}} \mu_{\mathrm{i}}^{2}-\sum_{\mathrm{k}}\left(\sum_{\mathrm{i}} \mu_{\mathrm{i}} \pi_{\mathrm{i} \mid \mathrm{k}}\right)^{2} \frac{\mathrm{N}_{\mathrm{k}}}{\mathrm{N}}\right) .
\end{aligned}
$$

Der Schichtungseffekt (4) ist bei identischen Umfängen $\mathrm{n}_{\mathrm{S}}$ und $\mathrm{n}_{\mathrm{gS}}$ dann gleich null, wenn in der Basisstichprobe kein Schichtungseffekt erzielt wird, wenn also für alle $\mathrm{k}$ Schichten $\pi_{\mathrm{i} \mid \mathrm{k}}=\pi_{\mathrm{i}}$ ist. Für diesen Fall gilt nun:

$$
\sum_{\mathrm{k}}\left(\sum_{\mathrm{i}} \mu_{\mathrm{i}} \pi_{\mathrm{i} \mid \mathrm{k}}\right)^{2} \frac{\mathrm{N}_{\mathrm{k}}}{\mathrm{N}}=\mu^{2}
$$

und damit wird die Varianz (3) wieder zu:

$$
\operatorname{Var} \overline{\mathrm{x}}_{\mathrm{gS}, \mathrm{S}}=\frac{1}{\mathrm{n}_{\mathrm{gS}}} \sum_{\mathrm{i}} \pi_{\mathrm{i}} \sigma_{\mathrm{i}}^{2}+\frac{1}{\mathrm{n}_{\mathrm{gs}}} \sum_{\mathrm{i}} \pi_{\mathrm{i}}\left(\mu_{\mathrm{i}}-\mu\right)^{2},
$$

der Varianz des Mittelwertschätzers einer uneingeschränkten Zufallsauswahl des gleichen Umfanges $n_{\mathrm{gS}}$ wie die Auswahl nach geschätzten Schichtgrößen. Sind jedoch nicht alle $\pi_{\mathrm{i} \mid \mathrm{k}}=\pi_{\mathrm{i}}$, so besitzt die geschichtete Basisstichprobe einen positiven Schichtungseffekt. Denn es gilt in diesem Fall (hier ist Quatember, 1996, auf S. 55 oben zu korrigieren):

$$
\operatorname{Var}\left(\sum_{\mathrm{i}} \mu_{\mathrm{i}} \pi_{\mathrm{i} \mid \mathrm{k}}\right)=\mathrm{E}\left(\sum_{\mathrm{i}} \mu_{\mathrm{i}} \pi_{\mathrm{i} \mid \mathrm{k}}\right)^{2}-\mathrm{E}^{2}\left(\sum_{\mathrm{i}} \mu_{\mathrm{i}} \pi_{\mathrm{i} \mid \mathrm{k}}\right)>0
$$

und somit

$$
\mathrm{E}\left(\sum_{\mathrm{i}} \mu_{\mathrm{i}} \pi_{\mathrm{i} \mid \mathrm{k}}\right)^{2}>\mu^{2} .
$$

Daraus folgt nun:

$$
\sum_{\mathrm{k}}\left(\sum_{\mathrm{i}} \mu_{\mathrm{i}} \pi_{\mathrm{i} \mid \mathrm{k}}\right)^{2} \frac{\mathrm{N}_{\mathrm{k}}}{\mathrm{N}}>\mu^{2}
$$

und schließlich:

$$
\operatorname{Var} \overline{\mathrm{x}}_{\mathrm{gS}, \mathrm{S}}<\operatorname{Var} \overline{\mathrm{x}}_{\mathrm{Z}} \text {. }
$$

\section{Literatur}

QUATEMBER A. (1996). Schichten mit geschätzten Schichtgrößen. Österreichische Zeitschrift für Statistik, 25. Jahrgang, Heft 1, 43-58. 